Discrete Comput Geom 29:229-238 (2003)

DOI: $10.1007 / \mathrm{s} 00454-002-0741-\mathrm{z}$

Geometry

\title{
Anchored Hyperplane Location Problems
}

\author{
Anita Schöbel \\ Department of Mathematics, University of Kaiserslautern, \\ Postfach 3049, 67653 Kaiserslautern, Germany \\ schoebel@mathematik.uni-kl.de
}

\begin{abstract}
The anchored hyperplane location problem is to locate a hyperplane passing through some given points $\mathcal{P} \subseteq \mathbb{R}^{n}$ and minimizing either the sum of weighted distances (median problem), or the maximum weighted distance (center problem) to some other points $\mathcal{Q} \subseteq \mathbb{R}^{n}$

This problem of computational geometry is analyzed by using nonlinear programming techniques. If the distances are measured by a norm, it will be shown that in the median case there exists an optimal hyperplane that passes through at least $n-k$ affinely independent points of $\mathcal{Q}$, if $k$ is the maximum number of affinely independent points of $\mathcal{P}$. In the center case, there exists an optimal hyperplane which is at maximum distance to at least $n-k+1$ affinely independent points of $\mathcal{Q}$. Furthermore, if the norm is a smooth norm, all optimal hyperplanes satisfy these criteria. These results generalize known results about unrestricted hyperplane location problems.
\end{abstract}

\section{Introduction}

Approximating a set of given points $\mathcal{Q}$ in $\mathbb{R}^{n}$ by a linear function is known as the linear fit problem or the hyperplane location problem. The goal is to find a hyperplane (represented by a linear function) minimizing the sum of weighted distances to the points in $\mathcal{Q}$, or minimizing the maximum weighted distance to the points in $\mathcal{Q}$, respectively. As distance measure any norm is possible, but also gauges and metrics have recently been discussed [S3], [PC]. In this paper a restricted version of the hyperplane location problem-the so-called anchored hyperplane location problem —is analyzed, namely, the hyperplane is additionally forced to pass through some given points $p \in \mathcal{P}$.

Hyperplane location problems appear in several mathematical disciplines, where they have mainly been studied with the Euclidean and the rectangular distance. In robust statistics variants of the hyperplane location problem are known as absolute errors regression, median problems, $L_{1}$ regression, $L_{\infty}$ regression, and orthogonal/vertical 
$L_{1}$-fit or $L_{\infty}$-fit problems, respectively, depending on the type of distance measure and on the objective function used. Related investigations go back to the 18th century (see, e.g., [B]). A motivation of these problems is given in [RL]. In numerical mathematics, certain approximation problems, for example the approximation of a given function by a linear function, lead in a natural way to the same type of problems, see, e.g., [R]. In computational geometry, hyperplane location problems are known as linear $L_{1}$ or $L_{\infty}$ approximation problems. Here, in particular, the time complexity of the Euclidean variant of the problem was investigated by several authors, see e.g., $[\mathrm{KM}]$ and $\left[\mathrm{HII}^{+}\right]$. The two-dimensional version of the problem has also been studied in operations research, known as the line facility location problem which is a special case of location problems. Line location problems in the plane were first discussed in [W], and later by many other authors, e.g., [MN1], [MT], and [LW]. Recently, line and hyperplane location problems have also been discussed for distance measures apart from the Euclidean and the rectangular distance, namely, for block norms in [S1], arbitrary norms in [S2] and [MS1], smooth norms (see [MS2]), and also for gauges (see $[\mathrm{PC}]$ ).

If distances are measured by a norm, the main results for unrestricted hyperplane location problems are the following incidence criteria. There always exists an optimal hyperplane for the median problem that passes through $n$ affinely independent points of $\mathcal{Q}$, and, in the center case, there always exists an optimal hyperplane which is at maximum distance from $n+1$ affinely independent points of $\mathcal{Q}$. This has first been noted for the Euclidean distance, independently by many authors (see, for example, [W], [MN1], [LW], [MN2], [KM], and [HII $\left.{ }^{+}\right]$) and has later been generalized to block norms and even to distances derived from arbitrary norms [S2], [MS1], [S3]. A slightly weaker condition for gauges in the case of the median objective function has recently been developed in [PC]. Furthermore, if and only if the norm is a smooth norm, all optimal hyperplanes in the median problem pass through $n$ affinely independent points of $\mathcal{Q}$, and in the center problem all optimal hyperplanes are at maximum distance from $n+1$ affinely independent points of $\mathcal{Q}$ [MS2].

In this paper a nonlinear programming technique is developed to transfer both incidence properties to restricted hyperplane location problems in the following sense. If the hyperplane approximating $\mathcal{Q}$ is forced to contain $k$ affinely independent points of a set $\mathcal{P}$, then there exists a median hyperplane passing through at least $n-k$ affinely independent points of $\mathcal{Q}$, and a center hyperplane which is at maximum distance from $n-k+1$ affinely independent points of $\mathcal{Q}$. Sharper results for smooth norms will also be

developed. For $k=0$ this directly yields analytical proofs for the incidence properties of unrestricted hyperplane location problems.

\section{The Anchored Hyperplane Location Problems}

Let $d$ be a distance derived from a norm, i.e.,

$$
d(x, y)=\gamma(y-x) \quad \text { for all } \quad x, y \in \mathbb{R}^{n}
$$

for some norm $\gamma$. We consider the following problems (AMH) and (ACH): 
Given two finite sets $\mathcal{P}$ and $\mathcal{Q}$ of points in $\mathbb{R}^{n}$, find a hyperplane $H$ passing through all points in $\mathcal{P}$ and minimizing

(AMH) $f(H)=\sum_{q \in \mathcal{Q}} w_{q} d(H, q), \quad$ or

(ACH) $g(H)=\max _{q \in \mathcal{Q}} w_{q} d(H, q), \quad$ respectively,

where $w_{q} \geq 0$ for all $q \in \mathcal{Q}$ are nonnegative weights, $d$ is a distance measure derived from a norm, and

$$
d(H, q)=\min _{x \in H} d(q, x) .
$$

Note that, although the results of this paper remain true for zero weights, points $q$ with weight $w_{q}=0$ can simply be deleted from $\mathcal{Q}$.

An optimal hyperplane $H$ for problem (AMH) is called an anchored median hyperplane, and an anchored center hyperplane is an optimal solution of problem (ACH).

First, note that the anchored hyperplane location problem is only feasible if there exists a hyperplane containing the whole set $\mathcal{P}$. In particular, $(\mathrm{AMH})$ and $(\mathrm{ACH})$ are feasible if and only if the maximum number of affinely independent points in $\mathcal{P}$ is smaller than or equal to $n$. Let $k=\operatorname{dim}_{\text {aff }}(\mathcal{P})$ denote the maximum number of affinely independent points in $\mathcal{P}$. Without loss of generality, we therefore assume in the following that

$$
k \leq n .
$$

Furthermore, note that if $k+\operatorname{dim}_{\mathrm{aff}}(\mathcal{Q}) \leq n$, then there exists a (clearly optimal) hyperplane containing all points in $\mathcal{P}$ and in $\mathcal{Q}$. To avoid this trivial case, we also assume that

$$
\operatorname{dim}_{\mathrm{aff}}(\mathcal{Q})>n-k .
$$

As mentioned before, both problems (AMH) and ( $\mathrm{ACH})$ have been studied extensively in their unrestricted versions, i.e., with $\mathcal{P}=\emptyset$ (see, e.g., [MS1], [S3], and the references therein). The restricted version of (AMH) has been discussed in [MN2] in a planar setting, i.e., $n=2$ and $\mathcal{P}$ consisting of one single point. For the Euclidean distance it has been shown that all optimal lines for problem (AMH) contain at least one point from the set $\mathcal{Q}$. The same has been noted in $[\mathrm{KM}]$.

In the following we generalize the incidence properties of unrestricted hyperplane location problems to the restricted versions of (AMH) and (ACH). The proofs we present combine the techniques developed in [S3] and in [PC]. They are based on the minimization of quasiconcave functions, which contain the sum, or the maximum of piecewise affine linear functions. Note that an affine linear function $f: \mathbb{R}^{n} \rightarrow \mathbb{R}$ is given by some vector $a \in \mathbb{R}^{n}$ and a real number $b$ such that $f(x)=\langle a, x\rangle+b$. If $b=0$ the function $f$ is linear. We first turn to the case of median hyperplanes.

\section{Anchored Median Hyperplanes}

Lemma 1. Let $\mathcal{M}=\{1,2, \ldots, M\}, M \geq n$, and let $h: \mathbb{R}^{n} \rightarrow \mathbb{R}^{+}$be a strictly positive and convex function, and $f_{m}: \mathbb{R}^{n} \rightarrow \mathbb{R}, m \in \mathcal{M}$, be affine linear and nonconstant 
functions. Consider the problem to minimize

$$
f(x)=\frac{1}{h(x)} \sum_{m \in \mathcal{M}}\left|f_{m}(x)\right| .
$$

Furthermore, suppose that a minimum of $f$ exists. Then there exists an optimal solution $x^{*}$ and a subset $\mathcal{M}^{*} \subseteq \mathcal{M}$ with $\left|\mathcal{M}^{*}\right| \geq n$ such that

$$
f_{m}\left(x^{*}\right)=0 \quad \text { for all } m \in \mathcal{M}^{*} .
$$

Moreover, if $h$ is strictly convex, all optimal solutions $x^{*}$ satisfy $f_{m}\left(x^{*}\right)=0$ for at least $n$ of the functions $f_{m}$.

Proof. The following set of hyperplanes

$$
H_{m}:=\left\{x \in \mathbb{R}^{n}: f_{m}(x)=0\right\}, \quad m \in \mathcal{M},
$$

partitions $\mathbb{R}^{n}$ into polyhedral (full-dimensional) cells. On each cell $C$ all the functions $\left|f_{m}\right|, m \in \mathcal{M}$, are affine linear, and therefore $\sum_{m \in \mathcal{M}}\left|f_{m}(x)\right|$ is also an affine linear function. Since $h$ is convex, $f$ is a quasiconcave function on each cell. Minimizing over each of the cells separately yields a minimum at a cell vertex (see, e.g., page 109, Theorem 3.5.3, of [BSS]) whenever it exists. Using that a finite optimal solution exists, a global optimum $x^{*}$ is then obtained as the best of all these minima. Hence, $x^{*}$ is also a cell vertex, yielding that it lies on the intersection of at least $n$ hyperplanes $H_{m}$. Defining

$$
\mathcal{M}^{*}:=\left\{m \in \mathcal{M}: x^{*} \in H_{m}\right\}
$$

shows the first result.

For the second part of the lemma, note that an affine linear function divided by a strictly convex function is a strictly quasiconcave function, meaning that on each cell $C$ the objective $f$ is strictly quasiconcave and therefore attains its minima only at cell vertices.

In the following we describe a hyperplane by its normal vector $s \in \mathbb{R}^{n} \backslash\{0\}$ and its intercept $b \in \mathbb{R}$, i.e.,

$$
H_{s, b}=\left\{x \in \mathbb{R}^{n}:\langle s, x\rangle+b=0\right\} .
$$

We can now state the main result for anchored median hyperplanes.

Theorem 1. Let $d$ be a distance derived from a norm $\gamma$, and let $k \leq n$ be the number of affinely independent points in $\mathcal{P}$. Then there exists an anchored median hyperplane passing through at least $n-k$ affinely independent points of $Q$.

Proof. First note that hyperplanes lying too far away from $\mathcal{Q}$ need not be considered, and since the length of the normal vector $s$ can be assumed to be bounded, we can restrict the problem to a compact feasible set yielding the existence of a minimum $s^{*}, b^{*}$. Furthermore, since $s \neq 0$ is required for a hyperplane, we conclude that the 
normal vector $s^{*}$ of an optimal solution to problem (AMH) has at least one nonzero component. Without loss of generality assume that this is the first one, i.e., $s_{1}^{*} \neq 0$. Dividing all coefficients by $s_{1}^{*}$ yields an optimal solution with the first component of the normal vector equal to 1 . Restricting the optimization problem (AMH) to hyperplanes with normal vectors $s$ satisfying $s_{1}=1$ hence yields the same optimal solution $s^{*}, b^{*}$. We will therefore additionally require $s_{1}=1$.

According to Mangasarian [M] or, independently, Plastria and Carrizosa [PC] the distance between a point $q \in \mathbb{R}^{n}$ and a hyperplane $H_{s, b}$ can be calculated by

$$
d\left(q, H_{s, b}\right)=\frac{|\langle s, q\rangle+b|}{\gamma^{0}(s)}
$$

where $\gamma^{0}$ denotes the dual (or polar) norm of $\gamma$, defined by

$$
\gamma^{0}(x)=\max \{\langle x, y\rangle: \gamma(y) \leq 1\} .
$$

For $\mathcal{P}=\left\{p_{1}, p_{2}, \ldots, p_{l}\right\}$ problem $(\mathrm{AMH})$ can now be reformulated as the following nonlinear programming problem in $n+1$ variables:

$$
\begin{aligned}
\min _{s, b} \frac{1}{\gamma^{0}(s)} \sum_{q \in \mathcal{Q}} w_{q}|\langle s, q\rangle+b| & \\
\text { such that }\left\langle s, p_{i}\right\rangle+b & =0 \text { for } i=1,2, \ldots, l, \\
s_{1} & =1 .
\end{aligned}
$$

Since $k$ is the maximum number of affinely independent points of $\mathcal{P}$ we conclude that the linear dimension

$$
\operatorname{dim}\left\{\left(\begin{array}{c}
p_{1} \\
1
\end{array}\right),\left(\begin{array}{c}
p_{2} \\
1
\end{array}\right), \ldots,\left(\begin{array}{c}
p_{l} \\
1
\end{array}\right)\right\}=k,
$$

where $\left(\begin{array}{c}p_{1} \\ 1\end{array}\right) \in \mathbb{R}^{n+1}, i=1,2, \ldots, l=|\mathcal{P}|$. Consequently, the coefficient matrix of

$$
\left\langle s, p_{i}\right\rangle+b=0 \quad \text { for } \quad i=1,2, \ldots, l
$$

has rank $k$, meaning that there exist $k$ variables which can be substituted and eliminated in the objective function. Together with $s_{1}=1$ (which is linearly independent of the equations $\left.\left\langle s, p_{i}\right\rangle+b=0\right)$ there remain $(n+1)-k-1=n-k$ variables, denoted by $t \in \mathbb{R}^{n-k}$. This leads to the following equivalent problem in $\mathbb{R}^{n-k}$ :

$$
\min f(t)=\frac{1}{\gamma^{0}(s(t))} \sum_{q \in \mathcal{Q}} w_{q}|\langle s(t), q\rangle+b(t)|
$$

with $s$ and $b$ affine linear functions,

$$
\begin{aligned}
& s: \mathbb{R}^{n-k} \rightarrow \mathbb{R}^{n} \quad \text { and } \\
& b: \mathbb{R}^{n-k} \rightarrow \mathbb{R} .
\end{aligned}
$$


Note that the $n$-vector $s(t)$ contains all components of the smaller vector $t$; hence the mapping $s$ is injective. Since the composition of a convex and an affine linear function still remains convex, and the functions $f_{q}(t)=w_{q}(\langle s(t), q\rangle+b(t))$ are affine linear we can apply Lemma 1 in dimension $n-k$ and conclude that there exists an optimal solution $t^{*}$ and a set $\mathcal{Q}^{*} \subseteq \mathcal{Q}$ with $\left|\mathcal{Q}^{*}\right| \geq n-k$ such that

$$
\left\langle s\left(t^{*}\right), q\right\rangle+b\left(t^{*}\right)=0
$$

for all $q \in \mathcal{Q}^{*}$. Defining $s^{*}=s\left(t^{*}\right)$ and $b^{*}=b\left(t^{*}\right)$ it follows that for all $q \in \mathcal{Q}^{*}$,

$$
q \in H_{s^{*}, b^{*}},
$$

meaning that the optimal hyperplane $H^{*}:=H_{s^{*}, b^{*}}$ passes through at least $n-k$ points in $\mathcal{Q}$.

It remains to show that $\mathcal{Q}^{*}$ can be chosen in such a way that $k=\operatorname{dim}_{\text {aff }}\left(\mathcal{Q}^{*}\right)=n-k$. To this end, let $\mathcal{Q}^{*}$ be the set of at least $n-k$ points of $\mathcal{Q}$ contained in an optimal hyperplane $H^{*}$. Define

$$
\begin{aligned}
& \overline{\mathcal{P}}:=\mathcal{P} \cup \mathcal{Q}^{*}, \\
& \overline{\mathcal{Q}}:=\mathcal{Q} \backslash \mathcal{Q}^{*},
\end{aligned}
$$

and note that each optimal solution (AMH) with $\overline{\mathcal{P}}$ and $\overline{\mathcal{Q}}$ is not worse than $H^{*}$ and hence also solves the original problem (AMH). If $\bar{k}=\operatorname{dim}_{\text {aff }}\left(\mathcal{Q}^{*}\right)<n-k$ we get that $\operatorname{dim}_{\text {aff }}(\overline{\mathcal{P}})<n$ and, due to the above result, an optimal solution $H^{\prime}$ exists passing through at least one more point of $Q$ than $H^{*}$. This procedure can be continued until the optimal hyperplane contains $n-k$ affinely independent points of $\mathcal{Q}$.

Two remarks should be added:

- Note that the optimal hyperplane may pass through more than $n-k$ affinely independent points since points in $Q$ that lie within the affine hull of $\mathcal{P}$ will automatically be covered.

- For $\mathcal{P}=\emptyset$ a halving property has been shown for locating a median hyperplane in normed spaces (see [S3]), i.e., if $Q^{+}(H), Q^{-}(H)$, and $Q^{0}(H)$ are the points of $Q$ lying below, underneath, and on the hyperplane $H$, respectively, then all median hyperplanes satisfy

$$
\left|\sum_{q \in Q^{+}} w_{q}-\sum_{q \in Q^{-}} w_{q}\right| \leq \sum_{q \in Q^{0}} w_{q} .
$$

In general, this property does not hold if $|\mathcal{P}| \geq 1$, not even for $n=2$ and the Euclidean distance, as the following example demonstrates:

Let $\mathcal{P}=\{0\}, \mathcal{Q}=\{(10,1),(11,0),(-10,1)\}$, and assume equal weights for the points in $\mathcal{Q}$. Then the optimal anchored line $l^{*}$ passes through 0 and $(10,1)$, with the two remaining points of $\mathcal{Q}$ lying on the same side of $l^{*}$. 


\section{Anchored Center Hyperplanes}

In this section, too, we first derive a result for minimizing a quasiconcave function. This time, we deal with a function $g$, which is given as the maximum of piecewise affine linear functions, all divided by the same convex denominator.

Lemma 2. Let $\mathcal{M}=\{1,2, \ldots, M\}, M>n$, and let $h: \mathbb{R}^{n} \rightarrow \mathbb{R}^{+}$be a strictly positive and convex function, and $f_{m}: \mathbb{R}^{n} \rightarrow \mathbb{R}, m \in \mathcal{M}$, be affine linear functions. Consider the problem to minimize

$$
g(x)=\frac{1}{h(x)} \max _{m \in \mathcal{M}}\left|f_{m}(x)\right| .
$$

Furthermore, suppose that a minimum of $g$ exists. Then there exists an optimal solution $x^{*}$ and a subset $\mathcal{M}^{*} \subseteq \mathcal{M}$ with $\left|\mathcal{M}^{*}\right| \geq n+1$ such that

$$
g\left(x^{*}\right)=\frac{\left|f_{m}\left(x^{*}\right)\right|}{h\left(x^{*}\right)} \quad \text { for all } \quad m \in \mathcal{M}^{*} .
$$

Moreover, if $h$ is strictly convex, all optimal solutions $x^{*}$ satisfy $g\left(x^{*}\right)=\left|f_{m}\left(x^{*}\right)\right| / h\left(x^{*}\right)$ for at least $n+1$ of the indices $m \in \mathcal{M}$.

Proof. First suppose that there exists some $x \in \mathbb{R}^{n}$ such that $f_{m}(x)=0$ for all $m \in \mathcal{M}$. Then $x$ is the optimal solution and the lemma is trivially true.

Otherwise, we can assume that $\max _{m \in \mathcal{M}}\left|f_{m}(x)\right|>0$ for all $x \in \mathbb{R}^{n}$. Define for all $m \in \mathcal{M}$ two cells given by

$$
\begin{aligned}
& C_{m}^{+}=\left\{x \in \mathbb{R}^{n}: f_{m}(x)=\max _{k \in \mathcal{M}}\left|f_{k}(x)\right|\right\}, \quad \text { and } \\
& C_{m}^{-}=\left\{x \in \mathbb{R}^{n}:-f_{m}(x)=\max _{k \in \mathcal{M}}\left|f_{k}(x)\right|\right\} .
\end{aligned}
$$

Then each of the $C_{m}^{+}, C_{m}^{-}$is either empty or a polyhedral set given by linear inequalities of type $f_{m}(x) \geq f_{k}(x)$ or $f_{m}(x) \leq-f_{k}(x)$ for all $k \in \mathcal{M}, k \neq m$. That is, the boundaries of the cells are given by the hyperplanes

$$
\begin{aligned}
& H_{k m}^{+}=\left\{x \in \mathbb{R}^{n}: f_{m}(x)=f_{k}(x)\right\} \text { and } \\
& H_{k m}^{-}=\left\{x \in \mathbb{R}^{n}: f_{m}(x)=-f_{k}(x)\right\}
\end{aligned}
$$

for $k \neq m$. On each cell, $\max _{k \in \mathcal{M}}\left|f_{k}(x)\right|$ is affine linear and, hence, $g$ is quasiconcave on each cell. Since a minimum exists, we conclude that there exists an optimal cell vertex $x^{*}$, e.g., of the cell $C_{\bar{m}}^{+}$(again, see [BSS]). Due to the assumption that $\max _{m \in \mathcal{M}}\left|f_{m}(x)\right|>0$ for all $x \in \mathbb{R}^{n}$, we know that $H_{k \bar{m}}^{+} \cap H_{k \bar{m}}^{-} \cap C_{\bar{m}}^{+}=\emptyset$ for all $k \in \mathcal{M}$ and hence each cell vertex of $C_{\bar{m}}$ is the intersection of $n$ of the hyperplanes $H_{k \bar{m}}^{+}, H_{k \bar{m}}^{-}, k \neq \bar{m}$, with pairwise different indices $k$. Consequently, the cardinality of

$$
\mathcal{M}^{*}=\left\{k \in \mathcal{M}: x^{*} \in H_{k \bar{m}}^{+} \text {or } x^{*} \in H_{k \bar{m}}^{-}\right\} \cup\{\bar{m}\}
$$

is greater than or equal to $n+1$, proving the first part of the lemma.

In case of a strictly convex function $h$, we get that $g$ is strictly quasiconcave on each cell $C_{m}$, and hence all optimal solutions are attained at cell vertices. 
Note that in [D] the following reduction result for minimizing convex functions $f_{m}$, $m \in \mathcal{M}$, has been provided: there exists an optimal solution $x^{*}$ and a subset $\mathcal{M}^{*} \subseteq \mathcal{M}$ with $\left|\mathcal{M}^{*}\right| \leq n+1$ such that $x^{*}$ is the optimal solution for the reduced problem

$$
\min \max _{m \in \mathcal{M}^{*}} f_{m}(x)
$$

i.e., also in this (opposite) case, an optimal solution can be found by looking only at subsets with cardinality of at most $n+1$.

Now the main result for finding anchored center hyperplanes can be shown.

Theorem 2. Let $d$ be a distance derived from a norm $\gamma$, and let $k \leq n$ be the number of affinely independent points in $\mathcal{P}$. Then there exists an anchored center hyperplane which is at maximum distance from at least $n-k+1$ affinely independent points of $Q$.

Proof. The proof works along the lines of the proof to Theorem 1. Assuming $s_{1}^{*}=1$ and using

$$
d\left(q, H_{s, b}\right)=\frac{|\langle s, q\rangle+b|}{\gamma^{0}(s)}
$$

for the distance between a point $q$ and a hyperplane $H_{s, b}$, (ACP) can be rewritten as

$$
\min g(t)=\frac{1}{\gamma^{0}(s(t))} \max _{q \in \mathcal{Q}} w_{q}|\langle s(t), q\rangle+b(t)|
$$

with $s$ and $b$ affine linear functions, $\gamma^{0}(s(t))$ convex, and $t \in \mathbb{R}^{n-k}$.

Applying Lemma 2 in dimension $n-k$ yields the existence of an optimal solution $t^{*}$ and a set $\mathcal{Q}^{*} \subseteq \mathcal{Q}$ with $\left|\mathcal{Q}^{*}\right| \geq n-k+1$ such that for $s^{*}:=s\left(t^{*}\right)$ and $b^{*}:=b\left(t^{*}\right)$ we get that

$$
g\left(H_{s^{*}, b^{*}}\right)=\frac{1}{\gamma^{0}\left(s^{*}\right)} \max _{q^{\prime} \in \mathcal{Q}} w_{q^{\prime}}\left|\left\langle s^{*}, q^{\prime}\right\rangle+b^{*}\right|=\frac{w_{q}}{\gamma^{0}\left(s^{*}\right)}\left|\left\langle s^{*}, q\right\rangle+b^{*}\right|
$$

for all $q \in \mathcal{Q}^{*}$. Hence, the optimal hyperplane $H^{*}:=H_{s^{*}, b^{*}}$ is at maximum distance from at least $n-k+1$ points in $\mathcal{Q}$, and using the same iterative argument as in the proof of Theorem 1 shows that these points can assumed to be affinely independent.

\section{Anchored Hyperplanes and Smooth Norms}

A smooth norm $\gamma$ is defined as follows. Consider the unit ball of $\gamma$, given by

$$
B_{\gamma}=\left\{x \in \mathbb{R}^{n}: \gamma(x) \leq 1\right\} .
$$

The norm $\gamma$ is called a smooth norm if $B_{\gamma}$ is supported by exactly one hyperplane for any point $x \in \partial B_{\gamma}$ on its boundary. In this case we get the following stronger result.

Theorem 3. Let d be a distance derived from a smooth norm $\gamma$, and let $k$ be the number of affinely independent points in $\mathcal{P}$. Then all anchored median hyperplanes pass through at least $n-k$ affinely independent points of $Q$, and all anchored center hyperplanes are at maximum distance from at least $n-k+1$ affinely independent points of $Q$. 
Proof. Since $\gamma$ is a smooth norm, the dual norm $\gamma^{0}$ is strictly convex, see, for example, $[\mathrm{K}]$. Consequently, we derive the following objective functions (analogously to the proof of Theorem 1), but with a strictly convex denominator $\gamma^{0}(s(t))$ :

$$
\min f(t)=\frac{1}{\gamma^{0}(s(t))} \sum_{q \in \mathcal{Q}} w_{q}|\langle s(t), q\rangle+b(t)|
$$

for the median problem, and

$$
\min g(t)=\frac{1}{\gamma^{0}(s(t))} \max _{q \in \mathcal{Q}} w_{q}|\langle s(t), q\rangle+b(t)|
$$

for the center problem, respectively. As in the proofs of Theorems 1 and 2, $s$ and $b$ are affine linear functions and $t \in \mathbb{R}^{n-k}$. Since the composition of a strictly convex function and an affine linear injective function still remains strictly convex we can apply the second parts of Lemmas 1 and 2, respectively, and conclude the result.

\section{Conclusion}

Theorems 1 and 2 provide the basics for polynomial-time algorithms to solve anchored hyperplane location problems in fixed dimensions with median or center objective function, since an enumeration approach is possible in both cases. If $k$ is the number of affinely independent points in $\mathcal{P}$ we have to check

- all $\left(\begin{array}{c}n-k \\ |\mathcal{Q}|\end{array}\right)$ hyperplanes passing through all points in $\mathcal{P}$ and through any (affinely independent) subset of $n-k$ points of $\mathcal{Q}$ in the median case, and

- all $\left(\begin{array}{c}n-k+1 \\ |\mathcal{Q}|\end{array}\right)$ hyperplanes which are at maximum distance from any (affinely independent) subset of $n-k+1$ points of $\mathcal{Q}$ and contain all points of $\mathcal{P}$ in the center case.

Since evaluating a hyperplane can be done in $O(|\mathcal{Q}|)$, this yields the following complexity results.

Corollary 1. If the distance has been derived from a norm, an anchored median hyperplane can be found in $O\left(|\mathcal{Q}|^{n-k+1}\right)$ time, and an anchored center hyperplane can be found in $O\left(|\mathcal{Q}|^{n-k+2}\right)$ time, assuming that a norm evaluation can be done in constant time. In the case that $d$ has been derived from a smooth norm, the same time complexity is sufficient to determine all optimal anchored hyperplanes.

\section{References}

[B] R.J. Boscovich. De litteraria expeditione per pontificiam ditionem, et synopsis amplioris operis, ac habentur plura ejus ex exemplaria etiam sensorum impressa. Bononiensi Scientiarum et Artum Instituto atque Academia Commentarii, 4:353-396, 1757.

[BSS] M.S. Bazaraa, H.D. Sherali, and C.M. Shetty. Nonlinear Programming, 2nd edition. Wiley, New York, 1993.

[D] Z. Drezner. On minimax optimization problems. Mathematical Programming, 22(2):227-230, 1982. 
$\left[\mathrm{HII}^{+}\right]$M.E. Houle, H. Imai, K. Imai, J.-M. Robert, and P. Yamamoto. Orthogonal weighted linear $L_{1^{-}}$and $L_{\infty}$-approximation and applications. Discrete Applied Mathematics, 43:217-232, 1993.

[K] G. Köthe. Topologische Lineare Räume, 2nd edition. Springer-Verlag, Berlin, 1966.

[KM] N.M. Korneenko and H. Martini. Hyperplane approximation and related topics. In J. Pach, editor, New Trends in Discrete and Computational Geometry, chapter 6, pages 135-162. Springer-Verlag, New York, 1993.

[LW] D.T. Lee and Y.F. Wu. Geometric complexity of some location problems. Algorithmica, 1:193-211, 1986.

[M] O.L. Mangasarian. Arbitrary-norm separating plane. Operations Research Letters, 24:15-23, 1999.

[MN1] J.G. Morris and J.P. Norback. A simple approach to linear facility location. Transportation Science, 14(1): $1-8,1980$.

[MN2] J.G. Morris and J.P. Norback. Linear facility location-solving extensions of the basic problem. European Journal of Operational Research, 12:90-94, 1983.

[MS1] H. Martini and A. Schöbel. Median hyperplanes in normed spaces—a survey. Discrete Applied Mathematics, 89:181-195, 1998.

[MS2] H. Martini and A. Schöbel. A characterization of smooth norms. Geometriae Dedicata, 77:173-183, 1999.

[MT] N. Megiddo and A. Tamir. On the complexity of locating linear facilities in the plane. Operations Research Letters, 1(5):194-197, 1982.

[PC] F. Plastria and E. Carrizosa. Gauge-distances and median hyperplanes. Journal of Optimization Theory and Applications, 110(1):173-182, 2001.

[R] J. Rice. The Approximation of Functions: The Linear Theory, Volume 1. Addison-Wesley, Reading, MA, 1964.

[RL] P.J. Rousseeuw and A.M. Leroy. Robust Regression and Outlier Detection. Wiley, New York, 1987.

[S1] A. Schöbel. Location least-distant lines with block norms. Studies in Locational Analysis, 10:139-150, 1996.

[S2] A. Schöbel. Locating least distant lines in the plane. European Journal of Operational Research, 106(1):152-159, 1998

[S3] A. Schöbel. Locating Lines and Hyperplanes-Theory and Algorithms. Number 25 in Applied Optimization Series. Kluwer, Dordrecht, 1999.

[W] G.O. Wesolowsky. Location of the median line for weighted points. Environment and Planning A, $7: 163-170,1975$

Received August 14, 2001, and in revised form July 8, 2002, and August 28, 2002.

Online publication December 20, 2002. 